\title{
Accuracy of Intraoperative Gross Examination of Myometrial Invasion in Stage I-II Endometrial Cancer
}

\author{
Prauk Sethasathien $^{1}$, Kittipat Charoenkwan $^{1 *}$, Sumalee Siriaunkgul $^{2}$
}

\begin{abstract}
Background: To assess the sensitivity, specificity, positive predictive value (PPV), negative predictive value (NPV), and accuracy of intraoperative gross examination (IGE) of uterine specimens in determining deep myometrial invasion and cervical invasion compared to final histology. Materials and Methods: The clinical, surgical and histological data of all FIGO stage I-II endometrial cancer (EC) patients who had primary surgery were reviewed. Results of the IGE for myometrial invasion and cervical invasion were compared to the final histology. The sensitivity, specificity, PPV, NPV, and accuracy of the IGE in determining deep myometrial invasion and cervical invasion were calculated. Association between clinico-pathological factors and discrepancy between IGE and final histology in the determination of myometrial invasion was also assessed. A $p$-value of $<0.05$ was considered significant. Results: From January 2007 to December 2012, 179 patients diagnosed with clinical stage I-II endometrial cancer underwent surgical staging. The sensitivity and specificity of IGE in detecting deep myometrial invasion were $42.4 \%$ and $90.0 \%$, respectively, and the PPV and NPV were $67.6 \%$ and $76.1 \%$. The overall accuracy of IGE was $74.3 \%$. The sensitivity and specificity of IGE in identifying cervical invasion were $28.6 \%$ and $97.5 \%$, respectively, while the PPV and NPV were $60.0 \%$ and $91.1 \%$. The overall accuracy of IGE was 89.4\%. Conclusions: The sensitivity of IGE for detecting deep myometrial invasion and cervical invasion in early-stage EC is too low to be used alone. Alternative methods including intraoperative frozen section analysis, preoperative three dimensional ultrasound, and preoperative magnetic resonance imaging should be strongly considered.
\end{abstract}

Keywords: Endometrial neoplasms - lymph node excision - myometrium - neoplasm staging

Asian Pac J Cancer Prev, 15 (17), 7061-7064

\section{Introduction}

Endometrial cancer (EC) is the most common gynecologic cancer in the United States. It is the fourth most common cancer found in women and the eight most common cause of death from cancer (Jemal et al., 2010; Zhu et al., 2012). EC is the second most common gynecologic cancer diagnosed worldwide following cervical cancer. At the time of diagnosis, the tumor is confined to the uterus (FIGO stage I-II) in approximately $80 \%$ of the cases. Therefore, these patients usually have good prognosis with five-year survival rate of up to $90 \%$ (Creasman et al., 2006; Wang et al., 2012; Balasubramaniam et al., 2013; Krusun et al., 2014). Surgery is generally considered standard primary treatment, which is followed by tailored postoperative adjuvant radiation and/or chemotherapy depending on surgical-pathological risk factors. The surgical procedures include total hysterectomy, bilateral salpingo-oophorectomy, and surgical staging. The practice of routine pelvic and paraaortic lymphadenectomy as parts of staging procedures remains debatable due to possible morbidities associated with the procedure without proven survival benefit. However, it is generally agreed that patients with any of these risk factors including serous or clear-cell histology, grade 3 tumor, tumor size $>2 \mathrm{~cm}$, deep myometrial invasion, and cervical invasion, would have a high-risk of nodal metastasis and should be candidates for lymphadenectomy (McMeekin et al., 2013). Unlike other risk factors, knowledge of myometrial invasion and cervical invasion is usually obtained intraoperatively. The accuracy of the method used to assess myometrial and cervical invasion is thus important for determining the need for lymphadenectomy. At our institution, intraoperative gross examination (IGE) of the uterine specimen has been employed for this purpose.

The objectives of this study were to assess the sensitivity, specificity, positive predictive value (PPV), negative predictive value (NPV), and accuracy of the IGE in determining deep myometrial invasion and cervical invasion in the uterine specimens compared to final histology.

\section{Materials and Methods}

After ethical approval, the clinical, surgical and histological data of all FIGO stage I-II endometrial cancer

${ }^{1}$ Department of Obstetrics and Gynecology, Faculty of Medicine, ${ }^{2}$ Department of Pathology, Faculty of Medicine, Chiang Mai University, Chiang Mai, Thailand*For correspondence:kittipat.c@cmu.ac.th, charoenkwan@live.com 


\section{Prauk Sethasathien et al}

patients who had primary surgery at our institution from January 2007 to December 2012 were reviewed. The patients who had any preoperative treatment and those with incomplete data were excluded. The main parameter of interest was myometrial invasion which was classified as confined to the endometrium, invaded to the innerhalf of the myometrium, and invaded to the outer-half of the myometrium. In addition, cancer invasion to the cervix, defined as presence of endometrial cancer below the level of internal cervical os, was examined. The final histological interpretation of myometrial invasion and cervical invasion by gynecologic pathologists was considered the gold standard. Results of the IGE for myometrial invasion and cervical invasion were compared to the final histology. The sensitivity, specificity, positive predictive value (PPV), negative predictive value (NPV), and accuracy of the IGE in determining deep myometrial invasion (invasion to the outer-half of the myometrium) and cervical invasion were calculated. Association between clinical-pathological factors including age, tumor size, histology, grade, and tumor location and discrepancy between IGE and final histology in the determination of myometrial invasion was also assessed.

Regarding the surgical procedures, vertical midline abdominal incision was employed in all cases. Upon entering the abdomen, peritoneal washing and thorough abdominal exploration was universally performed. Subsequently, total extrafascial hysterectomy and bilateral salpingo-oophorectomy were done. The uterus was then open vertically along the anterior wall from the fundus to the cervix using a scalpel. The depth of myometrial invasion and cervical invasion were then evaluated and noted by the operating surgeon. Intraoperative frozen section was not performed. The decision on whether or not to performed lymphadenectomy was made by the surgeon based on the findings of previously mentioned preoperative and intraoperative risk factors.

Statistical analysis was performed by using Stata ${ }^{\circledR}$ program version 12 (StataCorp LP, College Station, Texas, USA). The chi-square or Fisher's exact test, as appropriate, was used for an analysis of association between clinical-pathological risk factors and the IGE/ histology diagnostic discrepancy. The p-value of $<0.05$ was considered significant.

\section{Results}

From January 2007 to December 2012, 179 patients diagnosed with clinical stage I-II endometrial cancer underwent surgical staging procedures with available data on myometrial invasion. Median age was 55 years old (31-82). Most of the patients were 50 or older $(77.7 \%)$. Median tumor size was $3.0 \mathrm{~cm}(0.4-8.5)$. The majority of the patients had endometrioid histology $(85.4 \%)$ and grade 1 tumor $(62.3 \%)$. Tumor characteristics are demonstrated in Table 1.

Fifty-nine patients $(33.0 \%)$ had deep myometrial invasion from final histological evaluation. The sensitivity and specificity of IGE in detecting deep myometrial invasion were $42.4 \%$ (25/59) and 90.0\% (108/120), respectively. The PPV and NPV were $67.6 \%(25 / 37)$
Table 1. Tumor Characteristics ( $N=179)$

\begin{tabular}{llr}
\hline Characteristics & & No. $(\%)$ \\
\hline Surgical stage (FIGO 2009) & IA & $112(62.6)$ \\
& IB & $45(25.1)$ \\
Final histology & II & $22(12.3)$ \\
& Endometrioid & $152(85.4)$ \\
& Mucinous & $1(0.6)$ \\
& Serous & $1(0.6)$ \\
Final grade & Clear cell & $2(1.1)$ \\
& Mixed & $22(12.4)$ \\
Tumor location & 1 & $109(62.3)$ \\
& 2 & $37(21.1)$ \\
& 3 & $29(16.6)$ \\
& Fundus & $45(25.3)$ \\
& Body & $64(36.0)$ \\
& Lower segment & $9(5.1)$ \\
& Whole cavity & $60(33.7)$ \\
\hline
\end{tabular}

Table 2. Comparison of Myometrial Invasion Determined by Intraoperative Gross Examination Versus Final Histology

\begin{tabular}{lcrr}
\hline $\begin{array}{l}\text { Intraoperative gross } \\
\text { examination }\end{array}$ & \multicolumn{2}{c}{ Final histology } & Total \\
\hline Inner-half invasion & Outer-half invasion & \\
Outer-half invasion & $108(60.3 \%)$ & $34(19.0 \%)$ & $142(79.3 \%)$ \\
Total & $12(6.7 \%)$ & $25(14.0 \%)$ & $37(20.7 \%)$ \\
\hline
\end{tabular}

Table 3. Detailed Comparison of Myometrial Invasion Determined by Intraoperative Gross Examination Versus Final Histology

\begin{tabular}{lrrrr}
\hline $\begin{array}{l}\text { Intraoperative gross } \\
\text { examination }\end{array}$ & Endometrium & $\begin{array}{c}\text { Inner-half } \\
\text { invasion }\end{array}$ & $\begin{array}{c}\text { Outer-half } \\
\text { invasion }\end{array}$ & Total \\
\hline Endometrium & $15(8.4 \%)$ & $14(7.8 \%)$ & $6(3.4 \%)$ & $35(19.6 \%)$ \\
Inner-half invasion & $19(10.6 \%)$ & $60(33.5 \%)$ & $28(15.6 \%)$ & $107(59.8 \%)$ \\
Outer-half invasion & $0(0.0 \%)$ & $12(6.7 \%)$ & $25(14.0 \%)$ & $37(20.7 \%)$ \\
Total & $34(19.0 \%)$ & $86(48.0 \%)$ & $59(33.0 \%)$ & $179(100.0 \%)$ \\
\hline
\end{tabular}

Table 4. Association of Clinical-pathological Characteristics and Diagnostic Discrepancy between Intraoperative Gross Examination and Final Histology

\begin{tabular}{|c|c|c|c|}
\hline Characteristics & & $\begin{array}{l}\text { stic discrepancy } \\
\text { No }(\%)\end{array}$ & $\mathrm{p}$ value \\
\hline Age (years) & $\begin{array}{l}<50 \\
\geq 50\end{array}$ & $\begin{array}{r}8(20.0) \\
38(27.3)\end{array}$ & 0.35 \\
\hline Tumor size $(\mathrm{cm})$ & $\begin{array}{l}2 \mathrm{~cm} \text { cut-off point } \\
<2 \\
\geq 2\end{array}$ & $\begin{array}{r}6(26.1) \\
38(25.3)\end{array}$ & 0.94 \\
\hline Tumor size $(\mathrm{cm})$ & $\begin{array}{l}4 \text { cm cut-off point } \\
<4 \\
\geq 4\end{array}$ & $\begin{array}{l}23(23.2) \\
21(28.4)\end{array}$ & 0.44 \\
\hline Final histology & $\begin{array}{l}\text { Endometrioid } \\
\text { Mucinous } \\
\text { Serous } \\
\text { Clear cell } \\
\text { Mixed }\end{array}$ & $\begin{array}{rr}41 & (27.0) \\
0 & (0.0) \\
0 & (0.0) \\
1 & (50.0) \\
4 & (18.2)\end{array}$ & 0.72 \\
\hline Final histology & $\begin{array}{l}\text { rouping } \\
\text { Endometrioid } \\
\text { Non-endometrioid }\end{array}$ & $\begin{array}{r}41(27.0) \\
5(19.2)\end{array}$ & 0.41 \\
\hline Final grade & $\begin{array}{l}1 \\
2 \\
3\end{array}$ & $\begin{array}{r}27(24.8) \\
9(24.3) \\
9(31.0)\end{array}$ & 0.77 \\
\hline Tumor location & $\begin{array}{l}\text { Fundus } \\
\text { Body } \\
\text { Lower segment } \\
\text { Whole cavity }\end{array}$ & $\begin{array}{r}9(20.0) \\
15(23.4) \\
4(44.4) \\
18(30.0)\end{array}$ & 0.37 \\
\hline
\end{tabular}


Table 5. Comparison of Cervical Involvement Determined by Intraoperative Gross Examination Versus Final Histology

\begin{tabular}{|c|c|c|c|}
\hline \multirow{2}{*}{$\begin{array}{l}\text { Intraoperative gross } \\
\text { examination }\end{array}$} & \multicolumn{2}{|c|}{ Final histology } & \multirow[t]{2}{*}{ Total } \\
\hline & Yes & No & \\
\hline Yes & $6(3.4 \%)$ & $4(2.2 \%)$ & $(5.6 \%)$ \\
\hline No & $15(8.4 \%)$ & $154(86.0 \%)$ & $169(94.4 \%)$ \\
\hline Total & $21(11.7 \%)$ & $158(88.3 \%)$ & $179(100.0 \%)$ \\
\hline
\end{tabular}

and $76.1 \%(108 / 142)$, respectively. The overall accuracy of IGE was $74.3 \%$ (133/179). Table 2 and 3 compare myometrial invasion as determined by IGE to final histological diagnosis.

Discrepancy between IGE and final histology in the determination of myometrial invasion occurred in 46 cases $(25.7 \%)$. There was no significant association between clinical-pathological characteristics and the rate of diagnostic discrepancy (Table 4).

Cervical involvement by endometrial tumor was confirmed in 21 patients $(11.7 \%)$ from final histological assessment. The sensitivity and specificity of IGE in identifying cervical invasion were $28.6 \%(6 / 21)$ and $97.5 \%$ (154/158), respectively. The PPV and NPV were $60.0 \%(6 / 10)$ and $91.1 \%(154 / 169)$, respectively. The overall accuracy of IGE was 89.4\% (160/179) (Table 5).

\section{Discussion}

Lymphadenectomy has been considered an important part of the complete staging surgery for EC, which provides information on cancer spread, the need for postoperative adjuvant treatment, and prognosis (McMeekin et al., 2013). However, the procedure could result in prolonged operative time, increased blood loss, injury to adjacent structures including urinary tract and nerve, postoperative ileus, thromboembolism, lymphocyst, and lymphedema (Morrow et al., 1991; Orr et al., 1997). Therefore, the selective approach of lymphadenectomy has been adopted in many centers in an attempt to balance between the benefit of cancer spread information and the risk of complications associated with the procedure. With this approach, the pre-and intraoperative risk factors have been employed to determine the need for lymphadenectomy. Deep (outer-half) myometrial invasion, generally assessed intraoperatively, has been recognized as one of the important predicting factors for nodal metastasis (Creasman et al., 1987). Its presence usually indicated lymphadenectomy.

IGE has been proposed as a simple and inexpensive method to visually evaluate the depth of myometrial invasion (Doering et al., 1989). From the recent metaanalysis including 16 previous studies published during 1989-2011, the pooled sensitivity, specificity, PPV, NPV, and accuracy of IGE compared with final histology in detecting $\geq 50 \%$ myometrial invasion were $75 \%(95 \%$ confidence interval [CI] 72-78), 92\% (95\%CI 90-94), 80\% (95\%CI 76-84), 89\% (95\%CI 87-92), and 87\% (95\%CI 86-88), respectively (Mavromatis et al., 2012). These parameters were significantly higher compared to those of our study especially the sensitivity. The sensitivity of only $42.4 \%$ in this study suggested that IGE missed as many as $57.6 \%$ of patients who were candidates for lymphadenectomy based on the myometrial invasion criteria. Surprisingly, the sensitivity of IGE in identifying cervical invasion was even lower (28.6\%). These could be partly explained by the fact that IGEs were performed by surgeons with different level of expertise ranging from senior gynecology residents, gynecologic oncology fellows, to gynecologic oncologists. In addition, the anterior vertical technique used to open the uterus for intraoperative gross examination in this study was different from the lateral approach originally suggested, which is to cut open along the lateral uterine wall bilaterally to expose the anterior and the posterior wall and then make full-thickness transverse incisions through the tumor and myometrium for complete evaluation of myometrial invasion (Robboy et al., 1994). Furthermore, it has been noted that for certain endometrial tumors with infiltrative growth pattern, microscopic extent of myometrial invasion could be beyond that is grossly visible (McMeekin et al., 2013). Also, it was found that accuracy of estimation of myometrial invasion by gross examination decreased as tumor grade progressed, $87 \%$ in grade $1,65 \%$ in grade 2 , and $31 \%$ in grade 3 tumor (Goff and Rice, 1990). However, there was no significant association between clinical-pathological characteristics and the rate of diagnostic discrepancy between IGE and final histology found in this study.

The role of alternative methods for evaluating myometrial invasion has been explored. Intraoperative frozen section has been examined in the recent retrospective study including 816 patients with stage IA-IVB endometrial carcinoma (Turan et al., 2013). The consistency rate of intraoperative frozen section analysis and final pathological analysis in the evaluation of myometrial invasion was $85.4 \%$ with higher concordance in grade 2 and 3 than grade 1 tumor. The sensitivity and specificity of frozen section analysis in detecting $\geq 50 \%$ myometrial invasion were acceptably high, $88.8 \%$ and $98.3 \%$, respectively. In addition, many studies have demonstrated impressive validity of preoperative imaging technique for evaluating myometrial invasion in EC patients. The high accuracy of preoperative transvaginal three-dimensional ultrasound using VCI display (3D US VCI) for the assessment of myometrial invasion and cervical invasion was demonstrated in $40 \mathrm{EC}$ patients undergoing primary surgery (Jantarasaengaram et al., 2013). Comparing to final histology, the sensitivity, specificity, PPV and NPV of 3D US VCI in detecting deep myometrial invasion were $100 \%, 89.7 \%, 78.6 \%$, and $100 \%$, respectively. For prediction of cervical invasion, the sensitivity, specificity, PPV and NPV of 3D US VCI were $100 \%, 86.2 \%, 73.3 \%$ and $100 \%$, respectively. Similarly, the most recent systematic review including 442 patients from nine studies has demonstrated the high accuracy of magnetic resonance (MR) imaging for preoperative detection of deep myometrial invasion in endometrial cancer. The pooled sensitivity was $86 \%$ for both dynamic contrast-enhanced (DCE) and diffusion-weighted (DW) technique and the pooled specificity was $82 \%$ for the DCE and $86 \%$ for the DW technique (Andreano et al., 2014).

The strength of this study was that all the data were 


\section{Prauk Sethasathien et al}

extracted from a prospectively collected database of our division, designed specifically for this purpose. Therefore, the risk of recall bias and observer bias was minimized. However, the lack of collected information in certain aspects, especially on the level of the operator who examined the uterine specimen for each case, prevented us from reaching more meaningful conclusion regarding the contributing factors for the low accuracy of IGE in this study.

In conclusion, the sensitivity of IGE currently employed at our institution to detect deep myometrial invasion and cervical invasion in early-stage EC is too low to be used alone. Alternative methods including intraoperative frozen section analysis, preoperative three dimensional ultrasound, and preoperative MR imaging should be strongly considered.

\section{Acknowledgements}

Faculty of Medicine, Chiang Mai University and the National Research University Project under Thailand's Office of the Higher Education Commission provided funding support for this project.

\section{References}

Andreano A, Rechichi G, Rebora P, et al (2014). MR diffusion imaging for preoperative staging of myometrial invasion in patients with endometrial cancer: a systematic review and meta-analysis. Eur Radiol, 24, 1327-38

Balasubramaniam G, Sushama S, Rasika B, Mahantshetty U (2013). Hospital-based study of endometrial cancer survival in Mumbai, India. Asian Pac J Cancer Prev, 14, 977-80.

Creasman WT, Morrow CP, Bundy BN, et al (1987). Surgical pathologic spread patterns of endometrial cancer. A gynecologic oncology group study. Cancer, 60, 2035-41.

Creasman WT, Odicino F, Maisonneuve P, et al (2006). Carcinoma of the corpus uteri. FIGO $26^{\text {th }}$ annual report on the results of treatment in gynecological cancer. Int J Gynaecol Obstet, 95, 105-43.

Doering DL, Barnhill DR, Weiser EB, et al (1989). Intraoperative evaluation of depth of myometrial invasion in stage I endometrial adenocarcinoma. Obstet Gynecol, 74, 930-3.

Goff BA, Rice LW (1990). Assessment of depth of myometrial invasion in endometrial adenocarcinoma. Gynecol Oncol, 38, 46-8.

Jantarasaengaram S, Praditphol N, Tansathit T, Vipupinyo C, Vairojanavong K (2013). Three-dimensional ultrasound using volume contrast imaging (VCI) display for the preoperative assessment of myometrial invasion and cervical involvement in endometrial cancer. Ultrasound Obstet Gynecol, 43, 569-74

Jemal A, Siegel R, Xu J, Ward E (2010). Cancer statistics, 2010. CA Cancer J Clin, 60, 277-300.

Krusun S, Pesee M, Rasio W, et al (2014). Survival rate of early stage endometrioid adenocarcinoma of endometrium treated at Srinagarind hospital. Asian Pac J Cancer Prev, 15, 2217-20.

Mavromatis ID, Antonopoulos CN, Matsoukis IL, et al (2012). Validity of intraoperative gross examination of myometrial invasion in patients with endometrial cancer: a metaanalysis. Acta Obstet Gynecol Scand, 91, 779-93.

McMeekin DS, Yashar C, Campos SM, Zaino RJ (2013). Corpus: epithelial tumors. In: Barakat RR, Berchuck A, Markman M,
Randall ME, editors. Principles and practice of gynecologic oncology. Sixth ed. Philadelphia: Lippincott Williams \& Wilkins, 661-714.

Morrow CP, Bundy BN, Kurman RJ, et al (1991). Relationship between surgical-pathological risk factors and outcome in clinical stage I and II carcinoma of the endometrium: a Gynecologic Oncology Group study. Gynecol Oncol, 40, 55-65.

Orr JW, Jr., Holimon JL, Orr PF (1997). Stage I corpus cancer: is teletherapy necessary? Am J Obstet Gynecol, 176, 777-88; discussion 88-9.

Robboy SJ, Kraus FT, Kurman RJ (1994). Gross description, processing, and reporting of gynecologic and obstetric specimens. In: Kurman RJ, editor. Blaustein's pathology of the female genital tract. Third ed. New York: SpringerVerlag, 1225-40.

Turan T, Oguz E, Unlubilgin E, et al (2013). Accuracy of frozen-section examination for myometrial invasion and grade in endometrial cancer. Eur J Obstet Gynecol Reprod Biol, 167, 90-5.

Wang XW, Zhong TY, Xiong YH, Lin HB, Liu QY (2012). Lack of association between the CYP1A1 Ile462Val polymorphism and endometrial cancer risk: a meta-analysis. Asian Pac J Cancer Prev, 13, 3717-21.

Zhu XL, Ai ZH, Wang J, Xu YL, Teng YC (2012). Weighted gene co-expression network analysis in identification of endometrial cancer prognosis markers. Asian Pac J Cancer Prev, 13, 4607-11. 